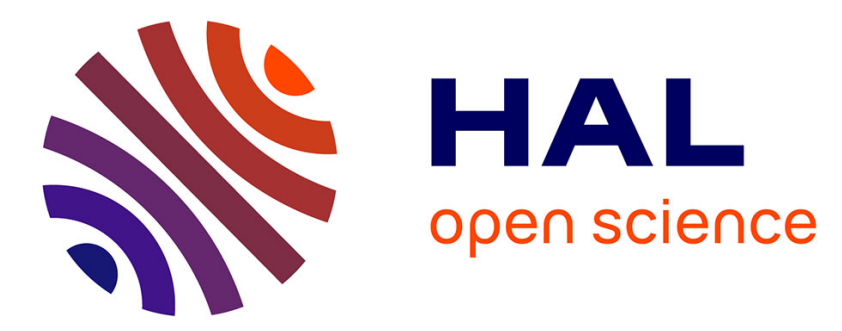

\title{
Foam trapping in a 3D porous medium: in situ obser- vations by ultra-fast X-ray microtomography
}

Raphaël Poryles, Nicolas Gland, Andrew King, Elisabeth Rosenberg, Loïc Barré, Thibaud Chevalier

\section{- To cite this version:}

Raphaël Poryles, Nicolas Gland, Andrew King, Elisabeth Rosenberg, Loïc Barré, et al.. Foam trapping in a 3D porous medium: in situ obser- vations by ultra-fast X-ray microtomography. Soft Matter, 2020, 16 (27), pp.6354-6361. 10.1039/d0sm00392a . hal-02955761

\section{HAL Id: hal-02955761 \\ https://hal-ifp.archives-ouvertes.fr/hal-02955761}

Submitted on 2 Oct 2020

HAL is a multi-disciplinary open access archive for the deposit and dissemination of scientific research documents, whether they are published or not. The documents may come from teaching and research institutions in France or abroad, or from public or private research centers.
L'archive ouverte pluridisciplinaire HAL, est destinée au dépôt et à la diffusion de documents scientifiques de niveau recherche, publiés ou non, émanant des établissements d'enseignement et de recherche français ou étrangers, des laboratoires publics ou privés. 


\title{
Journal Name
}

\section{ARTICLE TYPE}

Cite this: DOI: $00.0000 / x x x x x x x x x x$

\section{Foam trapping in a 3D porous medium: in situ obser- vations by ultra-fast $X$-ray microtomography}

\author{
Raphaël Poryles, ${ }^{* a}$ Nicolas Gland, ${ }^{a}$ Andrew King, ${ }^{b}$ Elisabeth Rosenberg, ${ }^{a}$ Loïc Barré ${ }^{a}$ \\ and Thibaud Chevalier ${ }^{* a}$
}

Received Date

Accepted Date

DOI: $00.0000 / x x x x x x x x x x$
One of the challenges in the study of foam transport in 3D porous media is having an adequate spatial and temporal resolution, to get a better understanding of the local phenomenon at the pore scale in a non-destructive way. We present an experimental study in which ultra fast XRay microtomography is used to characterize the foam trapping while the foam is flowing in a $3 \mathrm{D}$ porous medium. A preformed aqueous foam is injected into a rotating cell containing a 3D granular medium made of silica grains.

The use of rotating seals allows the cell to rotate continuously at the rate of one revolution per second, compatible with the fast X-ray tomography at SOLEIL synchrotron. We visualize the foam flow and track the trapping of bubbles with an acquisition time of about one second and a spatial resolution of a few microns (pixel size of one micron).

This allows us to extract characteristics and reliable statistics about trapped bubbles inside the granular medium and to observe their local behavior. With this setup and technique we access to the dynamics of foam trapping during the flow and the texture variations of the foam in the trapped zones. These local trapping events are well correlated with the macroscopical measurement of the pressure gradient over the cell.

\section{Introduction}

The optimization of oil recovery processes in reservoir rocks is a major issue considering the dominant part of hydrocarbons in the energy mix of today's world; in the coming decades, despite the acceleration of energy transition, hydrocarbons use will also remain substantial ${ }^{1}$. Typical oil production follows three stages: a primary recovery using a natural source of energy such as the pressure in the reservoir, a secondary recovery by injection of a fluid (gas or water flooding) to both maintain the pressure in the reservoir and sweep the oil towards production wells and finally a tertiary recovery, also called enhanced oil recovery or EOR, which involves a range of advanced physical and chemical methods to extract the most oil of the reservoir. Indeed, the first two production stages leave an important quantity of oil in the reservoir $(\approx 60 \%)^{233}$. To improve the final recovery, some studies investigate the usage of different surfactants solution to modify the interfacial tension between the liquid and oil phase $\mathrm{H}^{4}$, or the use of polymer solutions to increase the viscosity of the injected fluid.

\footnotetext{
a IFP Energies nouvelles, 1 et 4 avenue de Bois-Préau, 92852 Rueil-Malmaison, France ${ }^{b}$ Synchrotron SOLEIL, 91192 Saint-Aubin, France

* E-mail: raphael.poryles@yahoo.fr and thibaud.chevalier@ifpen.fr
}

Another prospective process is to use an aqueous foam, which is also a technique developped for decontamination processes ${ }^{5 / 6}$. Foam injection benefits lie in controlling the gas mobility by increasing its apparent viscosity during injection processes in order to mitigate the detrimental effects of low gas viscosity, reservoir heterogeneity and gravity override. It increases drastically the sweeping efficiency, from its high effective viscosity 7

At the pore-scale, foams are composed of metastable thin liquid films or "lamellae" and liquid carrying Plateau borders that split the gas phase into individual bubbles. The rheology of the foam is intimately related to the number of surfactant lamellae per unit volume, a parameter also called foam texture. Foam and gas trapping show a decrease of relative permeability which corresponds to an increase of apparent viscosity, resulting in a drop in mobility and a better efficiency $8+10$.

In order to understand and improve the efficiency of such foam flows, studies have been performed in a 2D Hele-Shaw cell, to observe the dynamics of the bubbles at the pore scale 11 .19. Micromodels experiments have shown mechanisms such as bubble trapping, fragmentation and coalescence ${ }^{11 / 12|16| 19}$. The trapping can be localized in a single pore or at the scale of inter connected pores $13[14$.

In the case of a 3D sample, most flow experiments are per- 


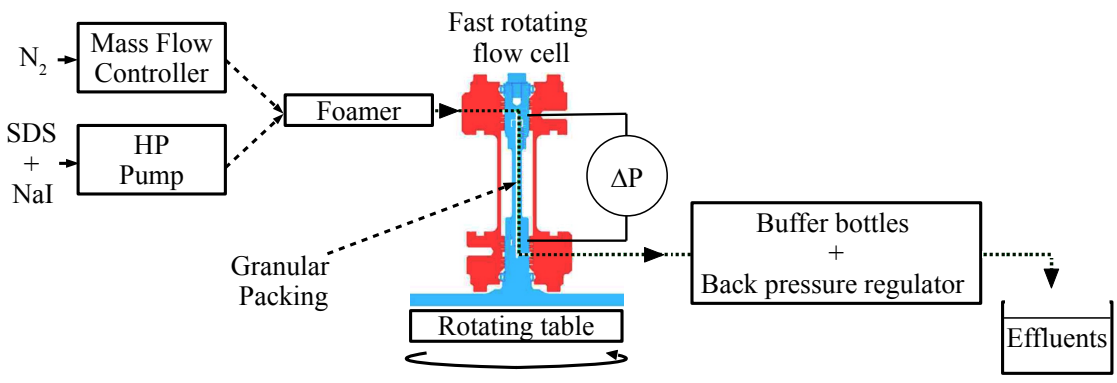

Fig. 1 Experimental setup. The foam is first generated by coinjection of a surfactant solution and nitrogen gas inside a first cell ("foamer"). It is then injected directly in the rotating flow cell filled with $\mathrm{SiC}$ grains under X-Ray beam, for tomographic measurement. A back pressure regulator connected to the cell outlet controls the system pressure. The differential pressure is measured between the inlet and the outlet of the rotating flow cell.

formed at the core scale, by measuring macroscopic values such as the differential pressure along the core which increases with the foam generation $20 \mid 22$. Studies have investigated various effects such as transport ${ }^{23}$, trapping and coalescence 2425 , hysteresis effects 26 27, as well as the nature of the gas and surfactant used 2829 .

Such measurements have also been coupled with 3D CT-scan imaging 10]30,35. Those studies have improved our understanding of foam flows in a porous medium, especially by imaging on large scales the porous medium during the flow 36 39. Limits of these works lie in the spatial resolution (they do not access to the dynamics of the bubbles at the pore scale). To reach the pore length scale, laboratory micro-tomography is an available technology, but it has a very low temporal resolution, and thus it is not adequate to study transient flows regimes $40 \mid 41$.

One possibility to couple high spatial and temporal resolution, is to image the fluid flow using ultra-fast synchrotron microtomography. Studies have been reported using this technology, generally applied to oil water systems in 3D porous media ${ }^{42 / 43}$. None of these studies have investigated foams in porous media. Only one study of a bulk foam flow by fast imaging has been reported 44 .

In this article, we image a flowing foam in a granular packing at high spatial $(1.1 \mu \mathrm{m})$ and temporal resolution $(1.5 \mathrm{~s})$, by using ultra-fast synchrotron X-Ray tomography. Using image analysis we show evidence of bubble trapping at the pore scale that give access to the texture variations of the foam in the trapped zones. It then allows us to find a direct link between the entrapment dynamics at the pore scale and the pressure variations at a core scale.

\section{Experimental setup and protocol}

\subsection{Experimental setup}

The experimental setup is presented in Figure 1 1 We use two different flow cells; the first one is used as a "foamer" to generate the foam that will be injected in the X-Ray imaging rotating cell. The foamer is a quartz cell of length $7 \mathrm{~cm}$ and internal diameter of $4.2 \mathrm{~mm}$. The rotating cell, where the observations are performed, is made of PEEK (polyetheretherketone), material which does not absorb much X-Rays, and has a length of $7 \mathrm{~cm}$ and an internal diameter of $2 \mathrm{~mm}$. Both cells are filled with silica grains of size distribution ranging from 112 to $150 \mu \mathrm{m}$. Mass measurement of the cells, gives us an estimation of the porosity of the packing $\phi$ close to $40 \%$ and a porous volume of $0.88 \mathrm{~mL}$. This porosity value is confirmed by image analysis of the tomographic volumes.

This rotating cell is fixed on an Hastelloy made support with rotating seals. This support is made of two parts, a rotating one (blue in Figure 1) and a static one (red in Figure 1). The blue part is screwed on a rotating table, while the red part is fastened to external support to be motionless. This allows the flow cell to rotate while the flow and pressure connections to the other parts of the experiment stay fixed. The sealing between the rotating part and the fixed part is ensured using rotating seals. The outlet of the cell is connected to two 1 liter buffer bottles. Pressure in the buffer bottles is controlled using a back pressure regulator (Brooks, SLA5820B). The effluents are collected after the back pressure regulator.

The different fluids injected during the experiment are the following. First a brine/surfactant solution of NaI at $5 \mathrm{~g} / \mathrm{L}$, and SDS (Sodium Dodecyl Sulfate) at $5 \mathrm{~g} / \mathrm{L}$. This solution is injected using a Vindum Pump (VP-12K). This high concentration of SDS ensure to be above the critical micellar concentration, and the NaI provides a better contrast in tomographic images. The second fluid is nitrogen gas $\left(\mathrm{N}_{2}\right)$, stored in a compressed gas cylinder and injected using a mass flow controller (Brooks, SLA5850S). To generate the foam, the surfactant solution and the $\mathrm{N}_{2}$ gas are directly coinjected at the inlet of the "foamer" using controlled flow rates.

\subsection{Experimental protocol}

Pressure measurements are performed at the inlet and outlet of the rotating cell, using absolute pressure sensors (Keller, PA-33X) from which the differential pressure over the cell $\Delta P$ is obtained. Pressure is acquired every second.

The tomography of the sample is performed using the PSICHE $\mathrm{X}$-Ray line at synchrotron SOLEIL in Saclay, France. The table has a rotating speed of one revolution in $3 \mathrm{~s}$ and the integration for our tomography is performed on half a rotation; the spatial resolution is $1.1 \mu \mathrm{m}$ in the three spatial dimensions. Acquired tomographic volumes have a size of $2000 \times 2000 \times 500$ voxels, allowing to observe the entire width of the cell $(2 \mathrm{~mm})$, on a height 
of $550 \mu \mathrm{m}$, which corresponds to several grains. During injection we acquire tomographic volumes at an imaging speed of $1.5 \mathrm{~s}$, every 40 seconds, during the entire course of the experiment.

We first inject a reference brine solution (NaI at $5 \mathrm{~g} / \mathrm{L}$ ) at different flow rates $q$ to measure the permeability $k$ of the grain packing in the rotating cell using steady-state method. By varying the flow rate $q$ and measuring the differential pressure $\Delta P$ over the cell, permeability is calculated by linear regression using Darcy law:

$$
q=\frac{k S}{\mu L} \Delta P
$$

with $S$ the cell's cross section, $L$ its length and $\mu$ the dynamic viscosity of brine $e^{45}$. The permeability of the rotating flow cell is $k=9.7$ darcy, with one darcy $\approx 0.99 \mu \mathrm{m}^{2}$, which is a unit used to characterize rocks permeability.

The two cells are then saturated with the surfactant solution by circulating several pore volumes. At this stage, we start coinjecting inside the foamer the surfactant solution and the gas. The surfactant solution is injected at a constant flow rate of $q_{w}=4.5 \mathrm{~mL} / \mathrm{hr}$, and the gas at a constant mass flow rate of 25.2 standard $\mathrm{mL} / \mathrm{hr}$. Considering that the pressure at the outlet of the cell is controlled by the back pressure regulator at $5 \mathrm{bar}$, the outlet gas flow rate at experiment conditions is gas $q_{g}=5.5 \mathrm{~mL} / \mathrm{hr}$. Those values were chosen with respect to preliminary tests in laboratory, so that the experiment lasts for a reasonable time, and gives an outlet foam quality $f g=q_{g} /\left(q_{g}+q_{w}\right)=0.55$. This 'wet' foam provides also a better contrast between the gas and bubbles flowing in the cell and the water near the grains. Foam is generated in the first cell and then flows through the rotating cell. This foam generation before the rotating cell limits entrance effect. We compute the different phase mean interstitial velocity as $v=q /(\phi . S)$, with $\phi . S$ the apparent surface of the cell. This gives us mean interstitial velocity $v_{w} \approx 0.99$ for the water and $v_{g} \approx 1.2 \mathrm{~mm} \cdot \mathrm{s}^{-1}$ for the gas.

\section{Observations}

\subsection{Pressure}

The differential pressure $\Delta P$ measured as a function of time is represented in Figure 2 The $\circ$ symbols on the figure represent times when tomographic images were performed. The experiment lasted 38000 seconds ( $\sim 10.5 \mathrm{hrs}$ ). We observe that the differential pressure $\Delta P$ gradually rises from 0 up to 9 bars after $30000 \mathrm{~s}$. It then stabilizes, and the experiment is in a stationary state. We observe strong fluctuations of the differential pressure during the foam invasion, reorganization and regeneration, with alternating phases of increase, and sudden drop of the pressure. Those fluctuations are due to trapping events of the bubbles inside the sandpack, followed by avalanche like processes, where the gas flows rapidly inside the cell. This behavior will be discussed with the support of the tomographic images in the following section.

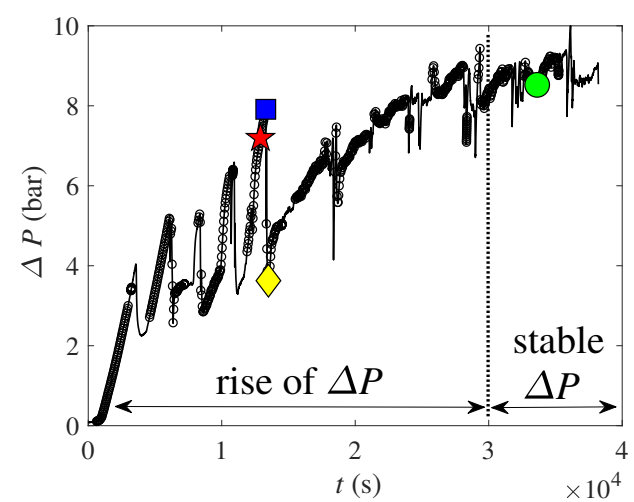

(b)

Fig. 2 Differential pressure $\Delta P$ in the rotating flow cell as a function of time $t$. The long time rise trend corresponds to the foam invasion, reorganization and regeneration inside the cell. The short time scale fluctuations are due to local trapping of gas bubbles. The continuous line is the pressure measured with the sensors, while the dots correspond to the times when tomographic images were acquired. The symbols correspond to 4 tomography that will be discussed with more consideration in the article.

\subsection{Tomography}

Tomographic sections of the sample under flow are given in Figure 3 . Figure 3 h represents a slice taken at mid-height of the tomographic volume. This image is an entire cross section of the $3 \mathrm{D}$ volume. We can see the grains (light gray) and the pores filled with fluids (dark gray to black). The zooms proposed in Figures 3 to 3 e reveal the detail of the distribution of the fluids in one selected area. Since the flow velocity is about $1 \mathrm{~mm} . \mathrm{s}^{-1}$, the section diameter $2 \mathrm{~mm}$ and the acquisition time $1.5 \mathrm{~s}$, the foam crosses several pores during an acquisition. This results in a kinetic blurring due to the rapid movement of bubbles and water in the pores and most of the pores appear to be completely filled with gas (in fact gas + water mixture) as in Figure $3 \mathrm{~b}$. However, intermittently, we can clearly distinguish spherical bubbles surrounded by water (with an intermediate gray level) as in Figure 3k. Since there is no blurring despite the high speed of the fluids, we believe that these bubbles are immobile and are representative of the trapped foam.

The sections shown in Figure 3 p to Figure 3 ere acquired at different times identified on the differential pressure curve given in Figure 2 The different colors correspond to the symbols showed in Figure $2(\star, \mathbf{m}, \bullet, \bullet)$.

Those four selected examples show significantly different behaviors. Figures $3 \mathrm{~b}$-d represent an event of strong $\Delta P$ variation during the foam generation (transient regime) and are taken in a short time interval (a few minutes), while Figures 3 e corresponds to the stationary regime. Hereafter, we detail the observations made in these four images.

The first one (Figure 3b, [Figure 2b $\star$ ]) corresponds to a high differential pressure level, just before a drop event. The bubbles are flowing inside the granular medium at a velocity of around $1 \mathrm{~mm} . \mathrm{s}^{-1}$ which is too high to image individually the bubbles. On the second one (Figure 3 ;, [Figure $2 \mathrm{~b} \mathbf{-}$ ) ), we can easily observe that bubbles are trapped inside the pores; this picture is taken just before the drop of differential pressure, when the entrapment is 

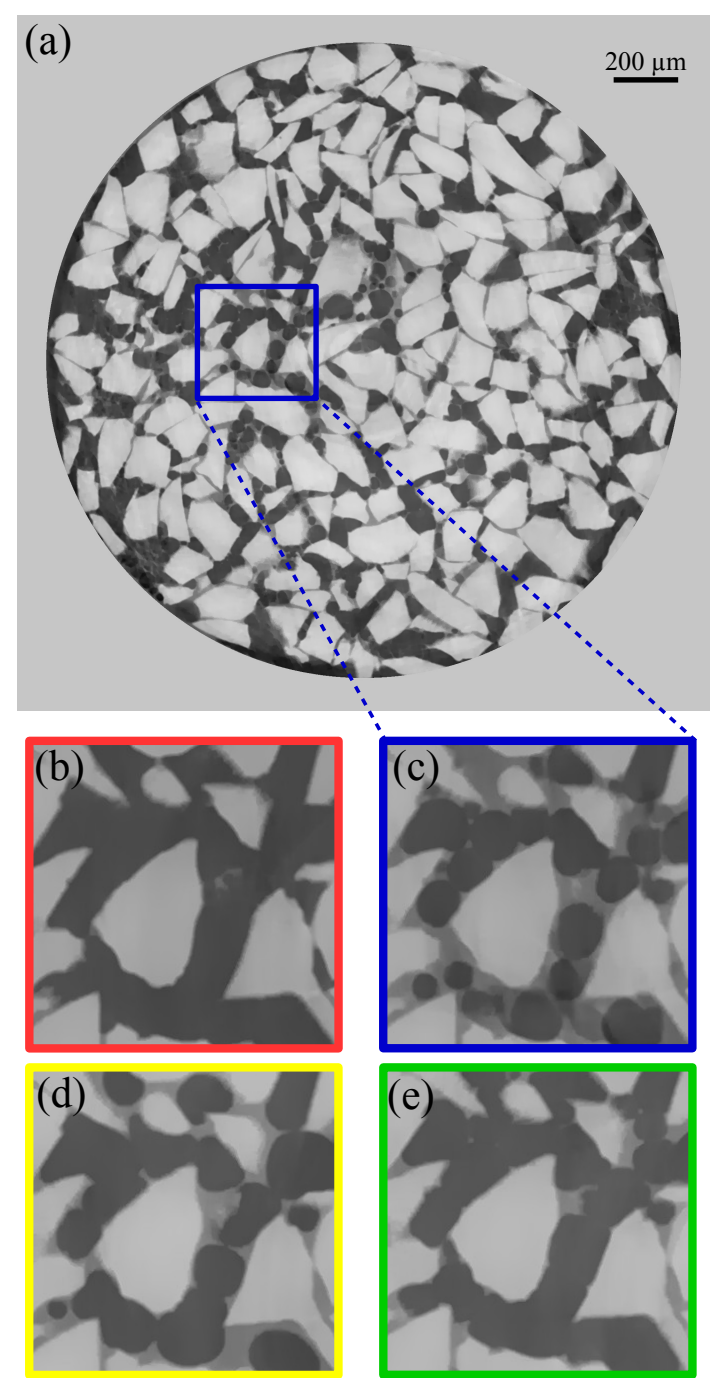

Fig. 3 Tomographic images examples.(a) Raw image corresponding of a full slice. (b-e) Zoom of the raw images at four different times of the experiment (b) Entire medium filled with flowing gas (c) Bubbles trapped at the differential pressure maximum peak (d) Residual bubbles trapped once the pressure drops after the peak (e) Stationary regime. These images corresponds to the symbols highlighted in Figure $2 \mathrm{p}$.

maximum. The third image (Figure $3 \mathrm{~d}$,[Figure $2 \mathrm{~b}\rangle$ ) represents the medium right after the drop. We observe that a number of bubbles are still trapped in the medium. Finally, the last picture (Figure 3e,[Figure 2p o] ) represents the medium in the stationary state at the end of the experiment. We observe that the packing is close to fully invaded by the flowing phase, but still shows some trapped bubbles in it. This will be discussed in Section 5 .

\section{Image analysis}

The image analysis is performed using Avizo software (Thermo Fisher Scientific). Our images have a high resolution and big size $(2000 \times 2000 \times 500)$, and we have a very large number of tomography over the entire experiment (700), which require to perform automated processing. The different steps of the treatment are presented in Figure 4. Starting from a raw image (Figure 3p), we first perform a phase segmentation. This segmentation is based on thresholding the histogram in three phases (gas phase, water phase and grain phase, Figure 43)). A post treatment is applied on this three phase segmented image, by filling the small holes inside a phase, and smoothing the edges of the different phases. Finally, we use a wathershed transformation to separate the different objects in a same phase $\frac{46}{}$. This allows us to separate the bubbles, by applying this separation on the gas phase made up of trapped and flowing bubbles, and to separate the pores by applying it to the gas + liquid phase. The different steps of the treatment are illustrated in Figure $4 \mathrm{p}$ for the pore separation and Figure 4. for the gas phase separation. Note that this analysis is performed in 3D (and not on a single slice), which increases highly the computation time. On Figure $4 \mathrm{p}$ and c, each color corresponds to a different label bubble/pore. We correlate each labeled pore with the labeled bubbles in it. By a connected component analysis, we obtain the volume of each pore, each bubble, their number in a single pore, and we correlate all those measurements together. Finally, Figure 5 shows a 3 dimensional reconstruction after the analysis, with the porous medium on the left, and only the gas phase on the right. This Figure demonstrates the complexity of the image treatment, which is not only performed slice by slice but has to take into account the three dimensional shape of the phases. Each color corresponds to different bubbles separated by our image analysis. (a)

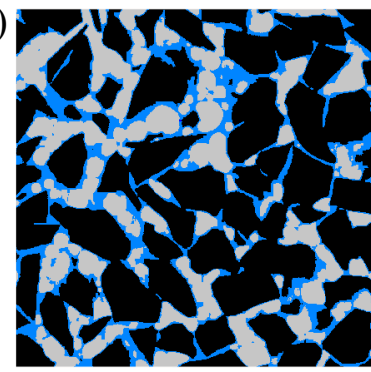

(c)

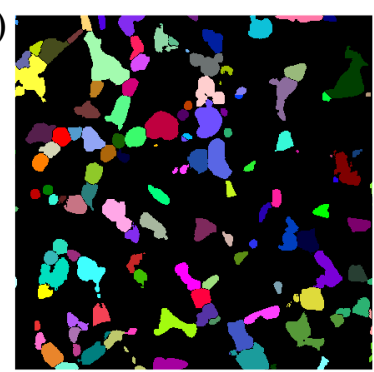

(b)

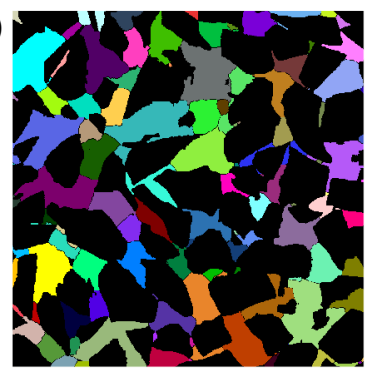

(d)

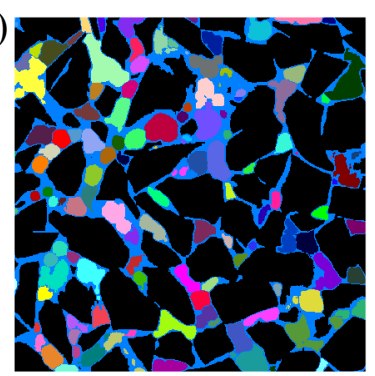

Fig. 4 Three steps of the image analysis, and final result. (a) Three phases segmentation (b) Pores separation; each color corresponds to a different labeled pore (c) Bubbles separation; each color corresponds to a different labeled bubble (d) Superposition of the labeled bubbles and the pore network. The description of the analysis is given in Section 4

\section{Results}

\subsection{Saturations values}

The first quantities that we obtain after image analysis are the values of gas and water saturations.

We define it as the percentage of pore occupation by the water 


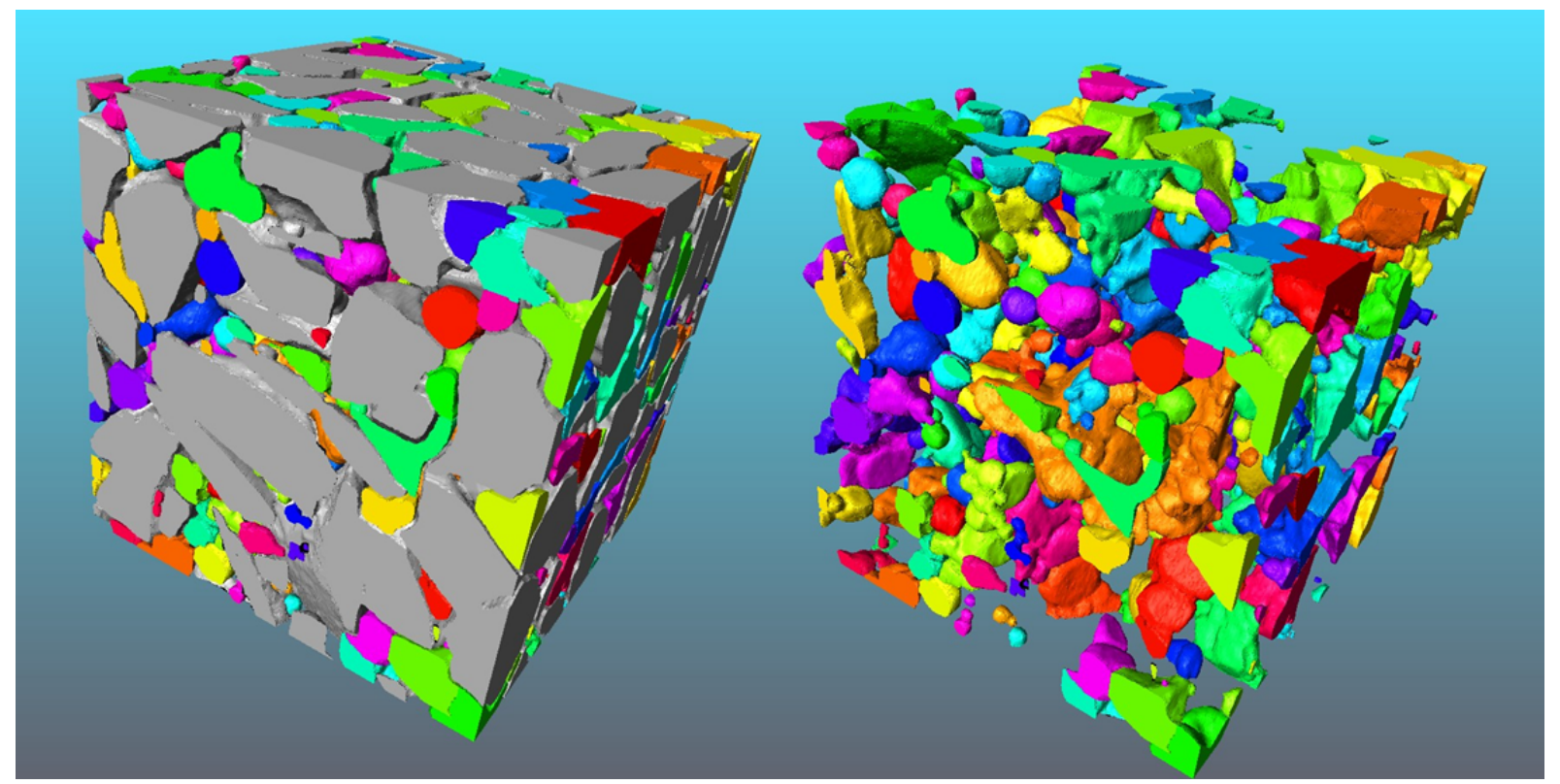

Fig. 5 Example of the tomographic reconstruction after image processing, with and without the solid phase (gray on the left image). The different colors corresponds to different bubbles separated by the image processing.

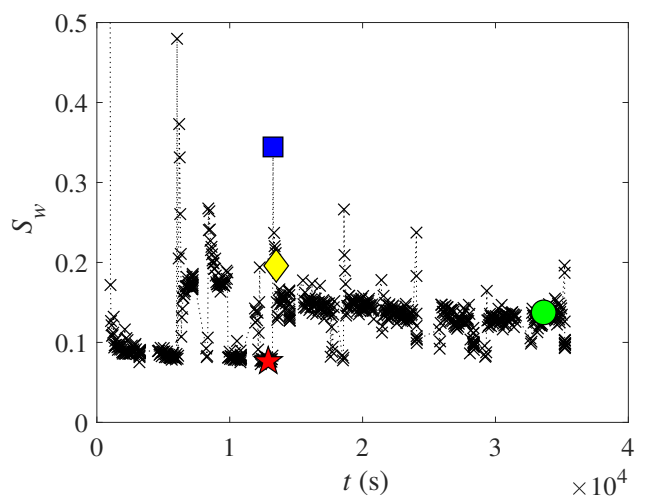

Fig. 6 Water saturation $S_{w}$ as function of time $t$. The colored symbols correspond to the examples in Figure 3

phase and the gas phase : $S_{g}=V_{g} / V_{p}$ and $S_{w}=V_{w} / V_{p}$, where $V_{g}, V_{w}$ and $V_{p}$ are respectively the gas, water and pore phase volumes (we have $S_{g}+S_{w}=1$, by definition). The variation of water saturation is presented in Figure 6 We observe that the average water saturation in the sandpack evolves from $10 \%$ up to $20 \%$ during the course of the experiment. This saturation is an apparent saturation and is smaller than the real water saturation since it does not take into account the flowing water in the flowing bubble phase.

Note that considering the high flow rates imposed in this experiment (equivalent to a few pores per second), even though the tomography is at a high speed (integration time of $1.5 \mathrm{~s}$ ), we cannot capture the flowing bubbles. This limitation implies that the observed bubbles (for instance in Figure 35) are necessarily trapped in the pore space (ie stopped or very slightly mobile) and that the phase identified as the flowing phase is actually constituted by bubbles and liquid in motion. In Figure 3月, we observe

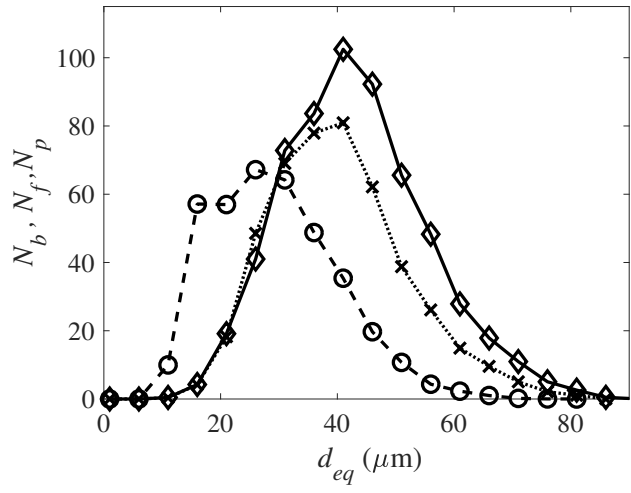

Fig. 7 Distribution of trapped bubbles [o], flowing bubbles $[x]$ and pores $[\diamond]$ equivalent diameter $d_{e q}$. The values $N_{b}, N_{f}, N_{p}$ is an average count over the entire experiment

that the porous medium is almost fully invaded, and no immobile bubble appear clearly in the pores which seem completely filled with gas. This is not physically the case and the bubbles are just moving too fast to be captured. The water is not visible when the foam is in motion and is only observable when associated with motionless trapped bubbles. The corollary benefit is that the observable water content becomes a marker of the trapping phenomenon and the dynamics of trapping can be tracked using the variations of $S_{w}$ as a function of time.

\subsection{Bubble size distribution}

Gas components labeling is presented on Figure 4 r and compared to the pore labeling in Figure 4 and $4 \mathrm{~d}$. The gas components separation is rather good but not perfect due to insufficient spatial resolution. We see some bubbles still attached to their neighbors (2 doublets of pink color in Figure 4k). At the end of this step 
aiming at identifying the gas components and the pores, the pores and the gas components can be matched. We saw previously that among these gas components, the bubbles in movement cannot be captured individually because of the dynamic blur and that these components occupy almost all of the pore. Their size is not significative of of a bubble one. On the other hand, the trapped bubbles are captured individually and the statistical analysis of their size and their evolution over time can be done, provided that they are separated from the moving bubbles. Since the sphericity could not be the criterion for selecting immobile bubbles, we used an empirical criterion which is that a separated gas component filling less than $70 \%$ of the pore is for sure a trapped bubble.

Following this criterion, we discriminate the "flowing bubbles" components that fill individually more than $70 \%$ of each pore and the "trapped bubbles", that are defined as the objects that fill individually less than $70 \%$ of each pore. This $70 \%$ value was chosen empirically, to get a large enough number of immobile bubbles, while having a good certainty in not selecting flowing bubbles. An important point is that trapped bubbles definition does not depend directly on the gas saturation but on the ratio of each bubble volume over the corresponding pore volume. For instance a pore could contain multiple bubbles summing up to over $70 \%$ of the pore volume. With this criterion we ensure to discard the bubbles flowing at high speed which would false the statistics since they are numerous and not representative of individual bubbles. Unfortunately this removes possible trapped bubbles filling more than $70 \%$ of a pore leading to an underestimation of the total number of trapped bubbles. This treatment is required to filter our data by removing the flowing bubbles (and a part of the trapped bubbles) enabling us to observe the trapping events.

These distributions computed over all the course of the experiment, including transient and stationary states, are presented in Figure 7 and compared to the pore size distribution. The equivalent diameter $d_{e q}$ is defined as $d_{e q}=\left(\frac{6 V}{\pi}\right)^{1 / 3}$.

Using those distributions, we evaluate the average pore diameter, which is about $40 \mu \mathrm{m}$, and the average trapped bubbles diameter around $25 \mu \mathrm{m}$. Obviously, the distribution of the flowing phase $N_{f}$ has no physical meaning since the temporal resolution does not capture the dynamic of this phase, which is a mixture of bubbles and water.

In the following, we will focus on the trapped bubbles, and disregard the moving phase.

\subsection{Bubbles number}

Figure $8 \mathrm{a}$ presents the number of trapped bubbles $N_{b}$ as a function of time. The behavior is very similar to the curve $S_{w}$ as a function of time (Figure 6). The colored symbols correspond to the examples in Figure 3 . The strong fluctuations are characteristic of the trapping events, as reported in Section 3.2 Figure $8 \mathrm{p}$ shows the number of trapped bubbles $N_{b}$ as a function of the water saturation $S_{w}$.

We see a very strong correlation between these two values, the drawn blue dashed line is a general trend for illustration. In both cases, the water saturation and the number of bubbles relate the events of foam trapping. $S_{w}$ variations illustrate the trapping dy-

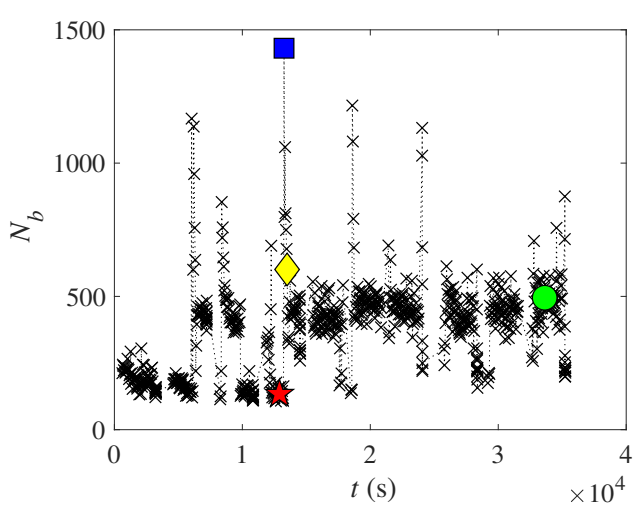

(a)

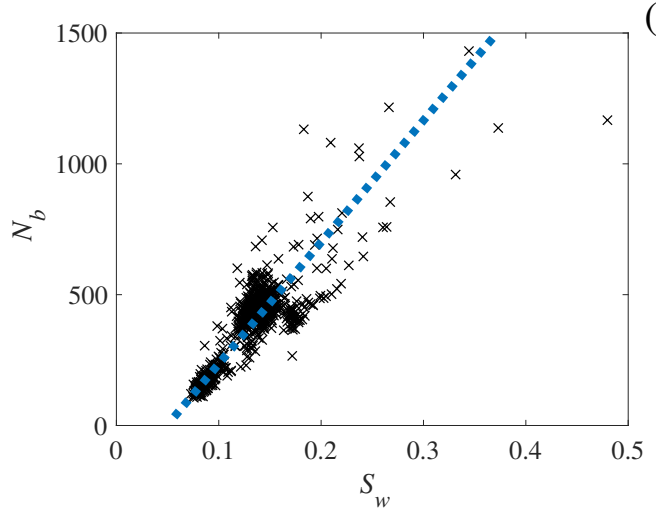

(b)

Fig. 8 (a) Number of trapped bubbles $N_{b}$ as a function time $t$. The colored symbols correspond to the examples in Figure 3 (b) Number of bubbles $N_{b}$ as a function of the water saturation $S_{w}$. The blue dashed line is a visual guide with no physical meaning.

namics since the water is not visible when the foam is in motion and is only observable when associated with motionless bubbles. Moreover, bubble number is directly related to the trapping since they are only accounted for when trapped otherwise they are counted as the flowing phase.

Additionally, we observe that the trapping is more important at the final steady state compared to the transient state at the beginning of the experiment. The foam stabilizes with time until it reaches the stationary state, while during transient state avalanche likes events are observed resulting from the rapid untrapping of bubbles and a bubble rearrangement inside the porous network.

\subsection{Pore occupation in trapped areas}

We can estimate the pore occupation number $P_{o}$, as the number of trapped bubbles divided by the number of pores occupied by these trapped bubbles $P_{o}=N_{b} / N_{p}^{o}$. This gives us in average how many bubbles are occupying each pore during trapping events. Figure 9 a shows the evolution of the pore occupation versus time. We see a global increase in the pore occupation. This evolves from 1.5 bubbles per pore up to 2.5 at the end of the experiment. The trend of this curve is very similar to the $\Delta P$ one. At the steady state, $P_{o}$ is bigger than two which means that we have more than one bubble per pore.

This is confirmed by the correlation between the pore occupa- 
tion number and the differential pressure in the cell $\Delta P$. Those results are presented in Figure 93. We observe that the pore occupation number increases with the pressure which confirms a foam texture refinement. When differential pressure $\Delta P$ increases, more bubbles are trapped, and the liquid compresses the bubbles. This key result directly connects a microscopic observation and an external macroscopic measurement of the pressure: it is one of the challenge to understand foam flow in porous medium and the origin of pressure variation.
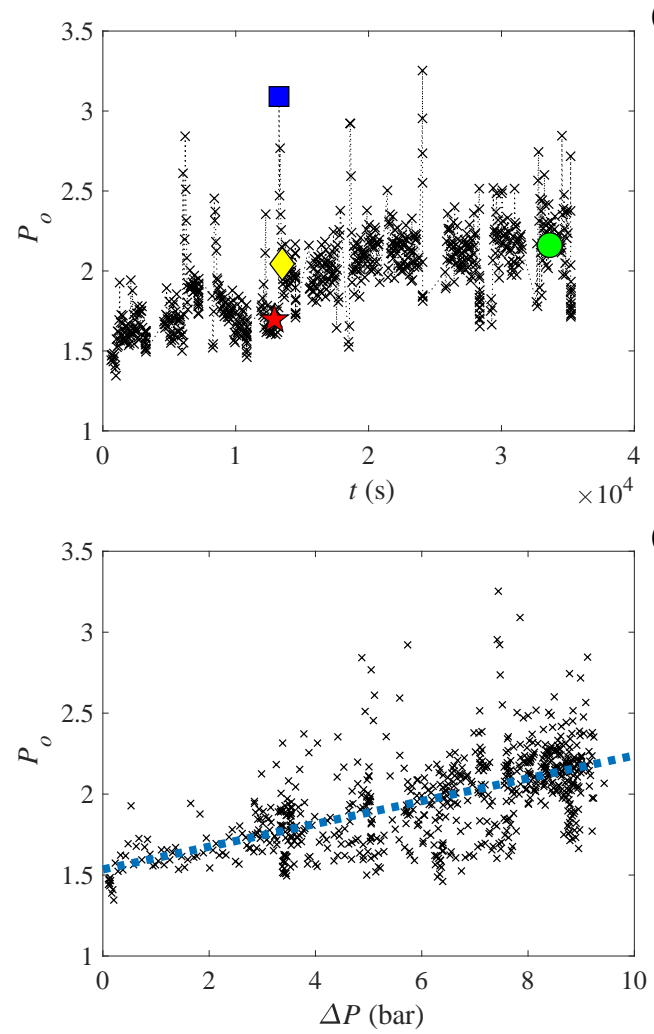

(b)

Fig. 9 (a) Pore occupation number $P_{o}$ as a function of time $t$. (b) Pore occupation $P_{o}$ as a function of the differential pressure $\Delta P$. The blue dashed line is a linear regression to illustrate the trend.

\section{Discussion and conclusion}

This study shows the potential of ultra-fast synchrotron tomography for foam flow in porous media studies. It is technically challenging, since the experiment is very long and the beam access time is usually short. Moreover, the spatial resolution $(1.1 \mu \mathrm{m})$ and the temporal resolution (1.5 s) were just enough to separate immobile bubbles and to track the foam trapping dynamics. It was not possible to image the moving foam since the flow rate was too important. The injection flow rate couldn't be reduced because of the limited beam access time. In those conditions, which represent the best compromise between acquisition and experimental conditions, we could acquire over several hundreds of tomographic 3D images (700 volumes) and obtain statistically representative and interesting new results.

We show that the erratic fluctuations in differential pressure $\Delta P$ are correlated to the foam trapping dynamics. Trapping events are associated with high frequency variations of the pressure gradient. A fast increasing/decreasing of the pressure is translated on the images by an increase of the number of bubbles and an increase of water saturation as the water is only observable when the movement of bubbles is stopped. Bubbles remobilization is evidenced by a low water saturation and a low number of bubbles on the images and a slow reincreasing of the pressure.

We show here that the in situ texture of the foam in non flowing areas is not constant and that it is refining as long as pressure increases to finally produce bubbles significantly smaller than the pore size.

This study shows the strong potential of ultra fast microtomography on synchrotron for studies of flow in 3D porous media and especially for the study of foam trapping. Future works could tune the different flow rates to obtain the 3D microscopic behavior in the many different flow regimes that are known ${ }^{12}$. Regimes to explore would include smaller injection rates, a longer rotating cell to avoid any entrance effects and a dryer foam. Those regimes would be closer with the existing literature cases of core flooding and their applications, but would also require much more time at a synchrotron facility.

An other approach would be to go towards direct applications, for instance by investigating the influence of oil on the foam invasion, and compare results to those obtained by neutron scattering 47 .

This work could be advantageously completed by a gas-tracer experiment using X-ray sensitive gas such as xenon or krypton to capture the regions with trapped gas. This would confirm our interpretation and validate our separation criterion between immobile gas and flowing gas.

\section{Conflicts of interest}

There are no conflicts to declare.

\section{Acknowledgements}

Experiments were performed on the "PSICHE" beamline at SOLEIL Synchrotron, France (proposal number 20180030). We are very grateful to the SOLEIL staff for smoothly running the facility. We also thank Samy Laabidi, Denis Grosjean, Rudy Albert and Chakib Ouali for the design of the rotating cell. The participants on the shifts were L. Barré, T. Chevalier, N. Gland, F. Lutz, R. Poryles and E. Rosenberg (alphabetical order).

\section{Notes and references}

1 IEA, World energy outlook: Outlook for oil, OCDE, Paris, 2019, pp. 129-173.

2 A. Muggeridge, A. Cockin, K. Webb, H. Frampton, I. Collins, T. Moulds and P. Salino, Phil. Trans. R. Soc. A, 2014, 372, 201220320.

3 R. Farajzadeh, A. Andrianov, R. Krastev, G. J. Hirasaki and W. R. Rossen, Adv. Colloid. Interfac., 2012, 183, 1-13.

4 A. Gbadamosi, R. Junin, M. Manan, A. Agi and A. Yussuf, Int. Nano Lett., 2019, 9, 171-202.

5 C. Dame, C. Fritz, O. Pitois and S. Faure, Colloid. Surface A, 2005, 263, 210-218. 
6 R. Deleurence, T. Saison, F. Lequeux and C. Monteux, Soft Matter, 2015, 11, 7032-7037.

7 H. Hemaptur, S. M. Mahmood, N. H. Nasr and K. A. Elraies, J. Nat. Gas Sci. Eng., 2018, 53, 163-180.

8 A. H. Falls, J. J. Musters and J. Ratulowski, SPE Reservoir Eng., 1989, 4, 155-164.

9 H. O. Lee, J. P. Heller and A. Hoefer, SPE Reservoir Eng., 1991, 6, 421-428.

10 M. Simjoo, Q. P. Nguyen and P. L. J. Zitha, Ind. Eng. Chem. Res., 2012, 51, 10225-10231.

11 B. Geraud, S. A. Jones, I. Cantat, B. Dollet and Y. Méheust, Water Resour. Res., 2016, 52, 773-790.

12 B. Geraud, Y. Méheust, I. Cantat and B. Dollet, Phys. Rev. Lett., 2017, 118, 098003.

13 S. A. Jones, N. Getrow and S. Vincent-Bonnieu, Soft Matter, 2018, 14, 3490-3496.

14 S. A. Jones, N. Getrow and S. Vincent-Bonnieu, Soft Matter, 2018, 14, 3497-3503.

15 A. A. Kellerm, M. J. Blunt and P. V. Roberts, Transp. Porous Media, 1997, 26, 277-297.

16 R. Liontas, K. Ma, G. Hirasaki and S. Biswal, Soft Matter, 2013, 9, 10971-10984.

17 K. Ma, R. Liontas, C. Conn, G. Hirasaki and S. Biswal, Soft Matter, 2012, 8, 10669-10675.

18 G. Ramadhan and Q. Nguyen, Transp. Porous Media, 2020, 131, 175-192.

19 I. Ben Salem, I. Cantat and B. Dollet, Colloid. Surface A, 2013, 438, 41-46.

20 P. Gauglitz, F. Friedmann, S. Kam and W. Rossen, Chem. Eng. Sci., 2002, 57, 4037-4052.

21 D. Huang, A. Nikolov and D. Wasan, Langmuir, 1986, 2, 672677.

22 A. Kovscek, D. Tretheway, P. Persoff and C. Radke, J. Petrol. Sci. Eng., 1995, 13, 75-86.

23 V. Prigiobbe, A. Worthen, K. Johnston, C. Huh and S. Bryant, Transp. Porous Media, 2016, 111, 265-285.

24 C. Portois, C. Boeije, H. Bertin and O. Atteia, Transp. Porous Media, 2018, 124, 787-801.

25 R. Thorat and H. Bruining, Transp. Porous Media, 2016, 112, 53-76.

26 S. Kahrobaei, S. Vincent-Bonnieu and R. Farajzadeh, Sci. Rep., 2017, 7, 8986

27 M. Lotfollahi, I. Kim, M. Beygi, A. Worthen, C. Huh, K. Johnston, M. Wheeler and D. DiCarlo, Transp. Porous Media, 2017, 116, 687-703.

28 E. Del Campo Estrada, H. Bertin and O. Atteia, Transp. Porous Media, 2015, 108, 335-354.
29 Y. Zeng, R. Farajzadeh, A. Eftekhari, S. Vincent-Bonnieu, A. Muthuswamy, W. Rossen, G. Hirasaki and S. Biswal, Langmuir, 2016, 32, 6239-6245.

30 S. Akin and A. R. Kovscek, Geol. Soc. Spec. Publ., 2003, 215, 23.

31 R. Farajzadeh, A. Andrianov, H. Bruining and P. Zitha, Ind. Eng. Chem. Res., 2009, 48, 4542-4552.

32 J. Lambert, I. Cantat, R. Delannay, A. Renault, F. Graner, J. Glazier, I. Veretennikov and P. Cloetens, Colloid. Surface. A, 2005, 263, 295-302.

33 Q. P. Nguyen, P. K. Currie, M. Buijse and P. L. J. Zitha, J. Petrol. Sci. Eng., 2007, 58, 119-132.

34 M. Simjoo and P. L. J. Zitha, Transp. Porous Media, 2015, 107, 799-820.

35 G. Tang and A. Kovscek, Transp. Porous Media, 2006, 65, 287307.

36 M. Amirmoshiri, Y. Zeng, Z. Chen, P. M. Singer, M. C. Puerto, G. Grier, R. Z. K. Bahrim, S. Vincent-Bonnieu, R. Farajzadeh, S. L. Biswal and G. J. Hirasaki, Energ. Fuel, 2018, 32, 1117711189.

37 A. Andrianov, R. Farajzadeh, M. M. Nick, M. Talanana and P. L. J. Zitha, Ind. Eng. Chem. Res., 2012, 51, 2214-2226.

38 A. Haugen, N. Mani, S. Svenningsen, B. Brattekås, A. Graue, G. Ersland and M. A. Fernø, Transp. Porous Media, 2014, 104, 109-131.

39 J. Tang, S. Vincent-Bonnieu and W. R. Rossen, Energy, 2019, 188, 116022.

40 M. Blunt, B. Bijeljic, H. Dong, O. Gharbi, S. Iglauer, P. Mostaghimi, A. Paluszny and C. Pentland, Adv. Water Resour., 2013, 51, 197-216.

41 J. Dunsmuir, S. Ferguson, K. D'Amico and J. Stokes, SPE, 1991, 22860.

42 S. Youssef, H. Deschamps, J. Dautriat, E. Rosenberg, R. Oughanem, E. Maire and R. Mokso, Int. Symp. SCA, 2013, 176, year.

43 M. Rücker, S. Berg, R. T. Armstrong, A. Georgiadis, H. Ott, A. Schwing, R. Neiteler, N. Brussee, A. Makurat, L. Leu, M. Wolf, F. Khan, F. Enzmann and M. Kersten, Geophys. Res. Lett., 2015, 42, 3888-3894.

44 C. Raufaste, B. Dollet, K. M. ans S. Santucci and R. Mokso, EPL, 2015, 111, 38004.

45 H. Darcy, Les fontaines publiques de la ville de Dijon. Exposition et application des principes à suivre et des formules à employer dans les questions de distribution d'eau, Dalmont, 1856.

46 S. Beucher and F. Meyer, Mathematical morphology in image processing, 1993, 34, 433-481.

47 R. Poryles, T. Chevalier, N. Gland, E. Rosenberg and L. Barré, Soft Matter, 2020, 16, 1771-1778. 